\title{
Femtosecond Pulse Light Filament-Assisted Microfabrication of Biodegradable Polylactic Acid (PLA) Material
}

\author{
Mangirdas MALINAUSKAS ${ }^{1}$, Laurynas LUKOŠEVIČIUS ${ }^{1}$, Simas BUTKUS ${ }^{1}$ and Domas PAIPULAS ${ }^{1}$ \\ ${ }^{1}$ Laser Research Center, Department of Quantum Electronics, Physics Faculty, Vilnius University, \\ Sauletekio Ave. 10, LT-10223 Vilnius, Lithuania \\ mangirdas.malinauskas@ff.vu.lt
}

\begin{abstract}
A novel method for efficient microfabrication in polylactic acid (PLA) using femtosecond pulse generated filaments is presented. Films and micro-woodpile structures of biodegradable plastic are laser engraved, drilled and cut through. The proposed technique is advantageous as the light filament ensures homogeneous energy concentration along the beam propagation axis, thus enables efficient energy delivery without axial translation of the sample. In comparison to sharp light beam focusing this method empowers avoidance of precise focal plane identification. In the experiment polylactic acid sheets and logs were immersed in water so the light filament is formed just above the processable material. The employed laser had a pulse width of $300 \mathrm{fs}$, average optical power of $5 \mathrm{~W}$, and operated at $1030 \mathrm{~nm}$ with $25 \mathrm{kHz}$ pulse repetition rate. This method was compared with traditional microfabrication using tight focusing by an 0.3 NA lens. Grooves, holes and various shapes were manufactured with a micrometer-scale precision as sample structures showing the simple implementation of the proposed novel approach. This method has a potential for finding numerous scientific and practical applications, among them micromechanics, microfluidics, cell growth experiments, and tissue engineering to mention as a few.
\end{abstract}

DOI: $10.2961 / \mathrm{j} 1 \mathrm{mn} .2015 .02 .0021$

Keywords: femtosecond pulses, light filament, laser microfabrication, polylactic acid, biodegradable polymer, microfluidics, tissue engineering.

\section{Introduction}

Direct Laser Writing (DLW) using ultrashort pulses has established itself as an advanced diverse material processing tool for micro-/nano-precise two-dimensional (2D) and threedimensional (3D) structures. It can be employed in both subtractive $[1,2,3]$ and additive manufacturing manners $[4,5,6]$ by applying organic and inorganic transparent materials as well as metals. Despite these recent technological advances, ultrashort pulses are still interesting for the scientific community as it allows structuring of various materials via non-linear light-matter interaction phenomena. For instance, during the last decade, light filamentation in water and other transparent media was studied in detail by several research groups [7, 8]. Yet it still remains an active topic for homogeneous energy distribution along the formed filament [9] as well as its potential practical application for rapid microfabrication of transparent materials [10]. Almost all materials can be processed with ultrashort laser pulses at high precision with $3 \mathrm{D}$ processing capabilities that including metals [11], dielectrics [12], as well as organic compounds [13]. Currently, metals are successfully fabricated both by surface texturing [14] and cutting in deeper trenches [15]. Though UV lithography employing masks can be efficiently used for 3D scaffolds manufacturing [16], it requires that the material is photosensitive for the applied irradiation wavelength which is a crucial experimental parameter [17]. Commonly, this is done by adding $0.5-2 \%$ w.t. of photoinitiator to the pre-polymer mixture. On the contrary, usage of fs pulses enable fabrication of optically transparent (non-photosensitized) materials, among them are elastomers [18]. Recently, a quasi-simultaneous mechanism of multiphoton absorbtion sequentially followed by avalanche ionization contributing to localized energy delivery resulting in cross-linking of the organic material was proposed [19]. Historically, plastics were drilled employing UV pulsed lasers [20], lately it was shown that welding of these types of materials can be achieved as well [21]. Beam shaping employing active (SLM and DMD [22, 23]) as well as passive (DOE or various masks [24]) techniques dramatically increase efficiency of some certain structures. Even straightforward changing of objectives can be advantageous in augmentation of the fabrication throughput [25]. Simultaneous additive and subtractive DLW manufacturing seems to be potential for improving the functionalities of the created structures which cannot be achieved with single step techniques [26]. Femtosecond pulses enable generation of real nanofeatures at surfaces [27] or small modified volumes within the bulk [28].

The material chosen here is PLA (PolyLactic Acid), which is already used for 3D DLW lithographic manufacturing for bio-related applications [29]. In general, polymers are attractive materials due to the possibility to incorporate them with functional non-linear materials [30], fluorescent dyes [31], and quantum dots [32]. It is worth mentioning that diverse laser-structurable polymers are biocompatible according to recent studies in vitro as well as in in vivo [33, 34]. The structures can be generated on substrates with different roughness and reflectivity [35, 36]. Micro-patterning of biodegradable polymers such as PolyCopraLactone (PCL) and PolyGlycolic Acid (PGA) using ultraviolet and femtosecond lasers was already reported previously [37]. Furthermore, surface modification of PCL membrane for tissue engineering applications was investigated [38]. At the same time, 


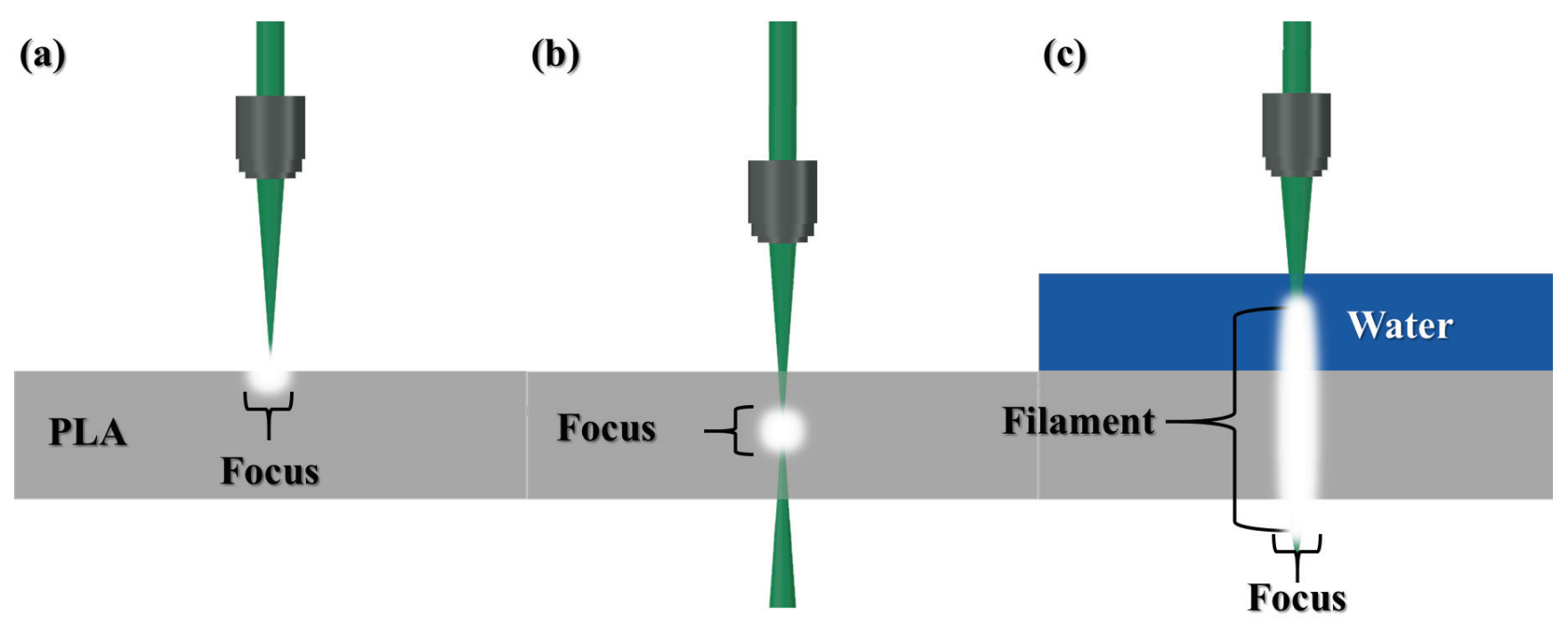

Fig. 1: Various laser beam focusing approaches: (a) - focused beam to the surface of transparent/opaque substrate; (b) - focused beam in the bulk of the transparent material; (c) - FPF in liquid assisted rapid microfabrication of transparent/opaque targets.

microfabrication of biodegradable materials for drug delivery remains an open issue [39]. Recently, near infra-red and visible fs pulses were applied for micro-precise processing of PLA [40].

In this technical communication, we report a study of FPF (Femtosecond Pulse Filament)-assisted microfabrication in PLA - from surface modification to hole drilling and curved shape cutting. The FPF method and its advantages are introduced and discussed over the classical focusing. Potential applications in biofluidics are presented by perforating sheets of plastic and 3D woodpile microstructures [41]. The experiments were realized with controllable and reproducible microprecision.

\section{Experimental procedure}

All the experiments were carried out using an $\mathrm{Yb}: \mathrm{KGW}$ femtosecond amplified laser system "Pharos" (Light Conversion Ltd.). The chosen parameters were as follows: average optical power $P=5 \mathrm{~W}$, wavelength - $1030 \mathrm{~nm}$, pulse width $300 \mathrm{fs}$, the repetition rate was set to $25 \mathrm{kHz}$. Two different strategies of microprecision fabrication were employed: one using DLW by tight focusing using linear translation stages, and the other using FPF employing loose focusing conditions (100 mm focal length $\mathrm{f}$ - theta lens) and galvanometric scanner. The samples were translated using "Aerotech" linear motion stages or the two axis galvanometric scanner ("ScanLab Inc.") were used for the beam deflection. All setup was controlled and the process was automated by a laser fabrication software, SCA (Altechna Ltd.). Figure 1 shows a schematic drawing of the light-matter interaction at sharp focussing and FPF cases, respetively. The laser beam was focused with an NA $=0.3$ and $10 x$ microscope objective (in a standard sharp focused beam fabrication) or $100 \mathrm{~mm}$-theta lens (for filament assisted fabrication) to the PLA samples or covering water layer, respectively. For the conventional focused beam fabrication the corresponding focused spot was of $\sim 4 \mu \mathrm{m}$ and the average laser power was adjusted depending on the specific exposure conditions and desired result. For the FPF the processable sample was immersed in water, the immersion depth was chosen to be approximately $0.5 \mathrm{~mm}$. Typical writing parameters for FPF were: focal spot diameter equal to $55 \mu \mathrm{m}$ and average laser power set to $5 \mathrm{~W}$. One can find more of detailed information regarding light filament assisted rapid fabrication elsewhere [10]. In case of focused beam fabrication, the sample was raster-scanned in X-Y plane followed by the change of the focusing plane in $\mathrm{Z}$ direction. In case of single holes it was just a translation along the $\mathrm{Z}$ axis. In the case of FPF only X-Y plane was raster scanned as the sample thickness was less than the formed filament length. All the objects were characterized by scanning electron microscopy "TM-1000" (Hitachi Ltd.) without adding any conductive layer. The clear PLA material was purchased from "Ultimaker BV" and the sheet samples were prepared by heating it up to $200^{\circ} \mathrm{C}$ and squeezing them between 2 glass slides. The micro-woodpile structure was 3D-printed using "Ultimaker" fused filament fabrication (FFF) 3D printer, the detailed protocol is described in [42]. In the presented study case clear as well as dyed (green and silver) PLA materials were used.

For the case of microscope objective focusing, filamentation does not occur due to insufficient pulse energy (the peak power of the pulse is below critical self-focusing limits). For the case of f-theta lens focusing, the pulse energy was set to approximately $200 \mu \mathrm{J}$, thus the corresponding peak power is exceeded by 100 fold relative to the critical power for self-focusing to occur. Due to the high pulse energy the length of the filament can reach up to $3 \mathrm{~mm}$ (depending on the focal position chosen) and therefore the cut can reach similar depth values. These values are both demonstrated theoretically and experimentally in $[10,43]$. The actual transverse dimensions of the filament are estimated to be less than $50 \mu \mathrm{m}$, however, due to the plasma coupling with the ablated target, the width of the cut varies depending on the material (due to better or worse plasma-target coupling). For PLA ablation the cut width is approximately $50-60 \mu \mathrm{m}$. This value remains approximately constant as the depth of the groove increases. 


\section{Results}

\subsection{Microfabrication with focused fs light beam}

Initially $\sim 200$ - $400 \mu \mathrm{m}$ sheets of PLA were microfabricated with a focused fs light beam. The films were drilled through by percussive drilling with continuous translation of the focus in $\mathrm{Z}$ direction as well as their surfaces were engraved by producing uniform straight line ridges.
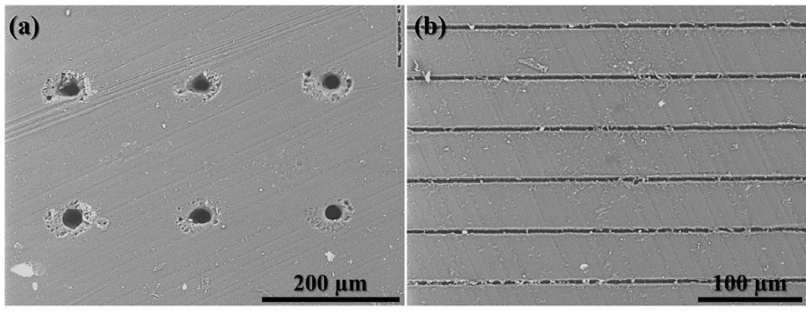

Fig. 2: Holes and grooves ablated in a PLA sheet. (a) The sample translation along the $Z$ axis was $0.5 \mathrm{~mm} / \mathrm{s}$ and $P=280$ $m W$. (b) The sample translation velocity perpendicular to the beam was $1 \mathrm{~mm} / \mathrm{s}$ and $P=10-15 \mathrm{~mW}$. The fabricated lines widths are $\sim 6 \mu \mathrm{m}$ and the affected zones widths are $\sim 11 \mu \mathrm{m}$.

The dimensions of the holes were chosen to be within the range corresponding to a single mammalian cell size. This was inspired by the currently active research vector of creating synthetic scaffolds by mimicking natural ones [44, 45]. Thus, a flexible and efficient method for the realization of $3 \mathrm{D}$ microarchitectures in biocompatible materials is of great interest $[46,47]$.

The produced microstructures are shown in Fig. 2. Laser processing parameters were chosen as follows: average optical power $P=5$ - $100 \mathrm{~mW}$, single pulse energy $E_{p}=0.2$ $4 \mu \mathrm{J}$ corresponding to $I=4.2-83 \mathrm{TW} / \mathrm{cm}^{2}$ (calculated according to the equation provided in [19]).

The obtained surface modifications are seen in Fig. 3. The groove width and depth increased as well as significant amount of debris was scattered around the structured microfeatures. Such surface modification are attractive for the enlargement of surface area and amplified wetting properties which are important for substrates and 3D scaffolds for cell culturing. The important note for such structuring is the ability to precisely tune the dimensions of the produced microfabrications by adjusting the employed laser power/exposure dose.

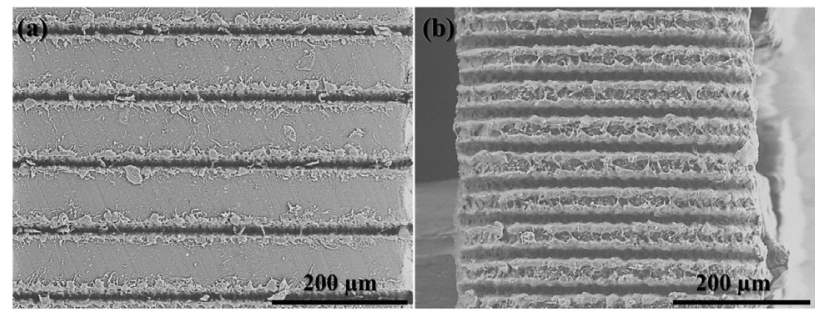

Fig. 3: Grooves ablated in a sheet and log. Sample translation was $1 \mathrm{~mm} / \mathrm{s}$ and $8 \mathrm{~mm} / \mathrm{s}, P=22 \mathrm{~mW}$ and $100 \mathrm{~mW},(a)$ and $(b)$, respectively. The fabricated grooves and affected zone widths in both cases equal to $\sim 15 \mu \mathrm{m}$ and $\sim 45 \mu \mathrm{m}$ respectively.
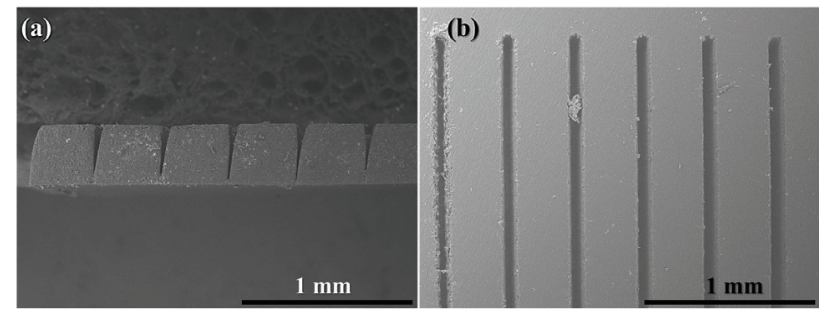

Fig. 4: Micro-grooves ablated in $350 \mu \mathrm{m}$ thick-PLA sheet by light filament assisted fabrication: (a) - side view, (b) - top view. The beam scan deflection (velocity with respect to the sample's surface) was changed from left to right as follows: 1 , $10,25,100,150$, and $250 \mathrm{~mm} / \mathrm{s}$ (only 5 are seen in (a) as the first one was totally cut off). The groove width is from $\sim 45$ $\mu \mathrm{m}$ to $\sim 65 \mu \mathrm{m}$, respectively. The area of the affected zone is almost a constant $95 \mu \mathrm{m}$.
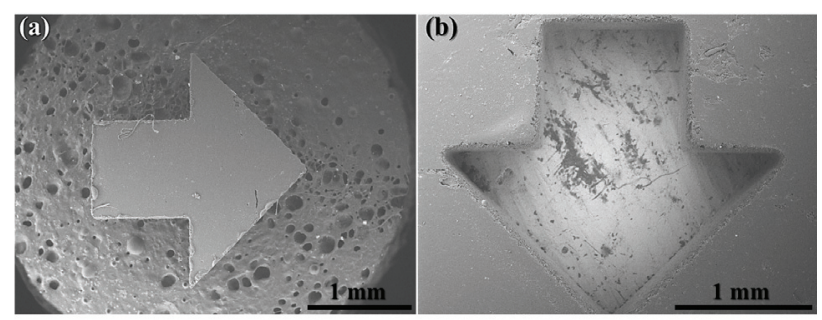

Fig. 5: An arrow cutout (a) and the remnant of the PLA sheet (b). The filament assisted cutting was performed at $100 \mathrm{~mm} / \mathrm{s}$ beam deflection velocity and the whole fabrication took $4 \mathrm{~s}$.

\subsection{Rapid fs pulsed light filament assisted fabrication}

For FPF fabrication the focusing conditions were altered. An f-theta lens was used, the average laser power was increased to $5 \mathrm{~W}$. This corresponded to a dramatic increase of $E_{p}=200 \mu \mathrm{J}$, yet a comparative $I=75 \mathrm{TW} / \mathrm{cm}^{2}$ at a $55 \mu \mathrm{m}$ diameter of the focused beam spot (described in details in [10]). Such intensity is well above the laser induced damage threshold for most of the photopolymers [48]. Anyhow, such cutting ensures high efficiency while keeping a rather tolerable incision smoothness (shown in Fig. 9).

As seen in Fig. 4 the obtained grooves are much deeper then their width as a result of filament induced cutting (the most left cut separated apart the sheet into separate pieces, actually it can be $>2 \mathrm{~mm}$ depth as was experimentally shown in [10]). Holes were drilled trough the films and through the woodpile structure's logs. For the micro-grooves and freeform pieces microfabrication the beam deflection velocity was set to be $v=1-100 \mathrm{~mm} / \mathrm{s}$.

Complex-shaped pieces: both positive and negative can be formed with this method. The positive ones can be cut out of the layer/bulk as the negative ones are have the same shape defect out of their volume (see Fig. 5). Functional microobjects such as microgears or arrows could be used for applications in biomechanics and micromechanics, both at the scientific as well as practical implementation level (see Fig. 5 - 8). 

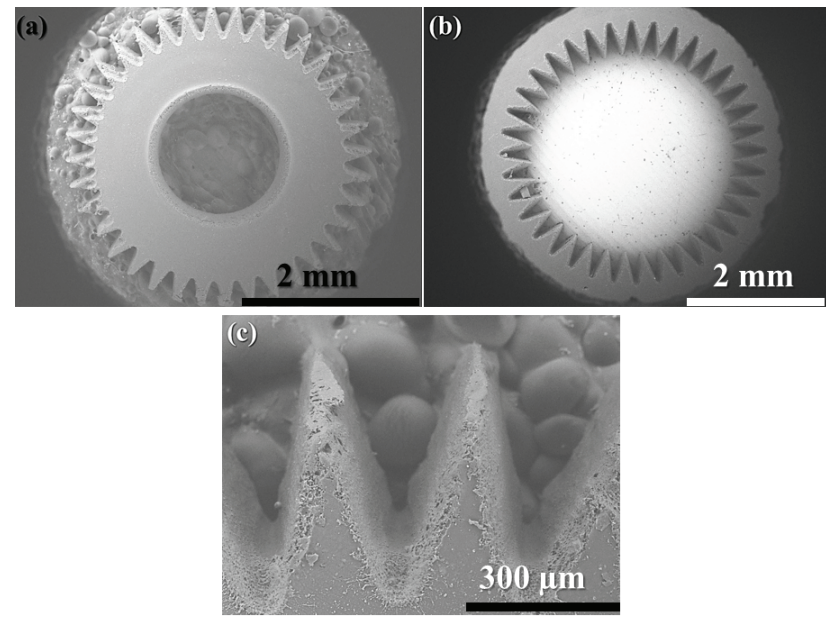

Fig. 6: A gear with microteeth cutout (a) and the remnant of the PLA sheet (b). (c) Is a magnified image of the microgears edge of (a). Beam deflection velocity $-100 \mathrm{~mm} / \mathrm{s}$, fabrication took 30 s. No additional specimen post-processing was performed.

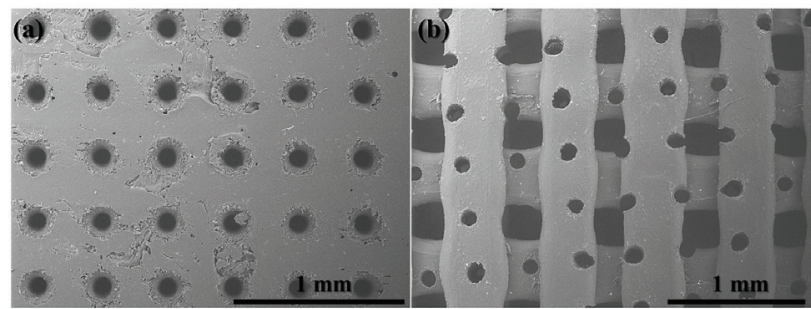

Fig. 7: Microholes produced in the PLA sheet (a) and the microwoodpile structure $(b)$. The uniform diameter is $\sim 100 \mu \mathrm{m}$ as the affected zone is slightly enlarged equal to $\sim 160 \mu \mathrm{m}$. (b) Is a backside view of the structure, thus the affected zone is negligible.

Optical density spectra of pure and colored PLA sheets were obtained with "UV Probe" ("Shimadzu") spectrophotometer (see Fig. 8 (a)). (b) - various shapes sample microstructures processed (engraved, drilled) or obtained (cut out) via femtosecond pulse sharp beam focussing and filament-assisted fabrication as seen by the naked eye. The reference squares corresponds to $1 \mathrm{~mm}$. Surprisingly, yet in spite of differently colored PLA there was no significant variation in laser processing parameters or deterioration in microfabricated sample quality as one could expect [49].

\section{Discussion}

In this report, we have experimentally demonstrated that that liquid-assisted fabrication has several hereinafter mentioned advantages. The main factor is its contribution as a media for filament formation which allows homogeneous energy distribution along the beam and ensures efficient energy delivery required for rapid microfabrication. Thus, with the current experimental conditions the light filament helps to avoid the precise matching of focal plane to the sample as well as $\mathrm{Z}$ translation for samples of $2 \mathrm{~mm}$ and more. Additionally, it helps remove the excess of accumulated heat and ablation generated microparticles which stay on the sample as debris (noticed in performed experiments, but not presented here). In the studied case, for the femtosecond pulse-generated light filament formation and its efficient application for rapid fabrication at employed irradiation conditions a 10-fold higher average power was used. It is noticeable that efficiency increment dependence on laser power is almost linear and has no significant threshold within the examined range (at least as long as the beam peak power is exceeded by several fold [10]). This offers higher power fs laser to be implemented for further increase of fabrication throughput. The proposed approach is practicable due to its disregard to the material filling ratio ( bulk or porous). The precise cut can be realized through all its thickness despite its micro-architecture as shown in Fig. 9

However, some drawbacks, such as sample submersion into the water should be taken into account. FPF requires a water reservoir, and in this case only $2.5 \mathrm{D}$ fabrication is possible. If curved surfaces are processed then additional $\mathrm{Z}$ axis positioning may become inevitable. Also, the absorption or irradiation wavelength in liquid cannot be neglected as it introduces energy losses and evaporation of the same liquid. Employment of frequency doubled (visible) wavelengths could be considered in the future experiments and targeted applications. Yet for the shorter wavelengths the self-action would be reduced and the non-linear absorbtion would be increased. Therefore in this requires additional theoretical and experimental investigation.

In the carried out experiments there was no significantly noticeable difference regarding the colors of the PLA material. Yet it is evident that for the visible wavelengths it would alter the fabrication results, thus power adjustment for each color would be necessary.
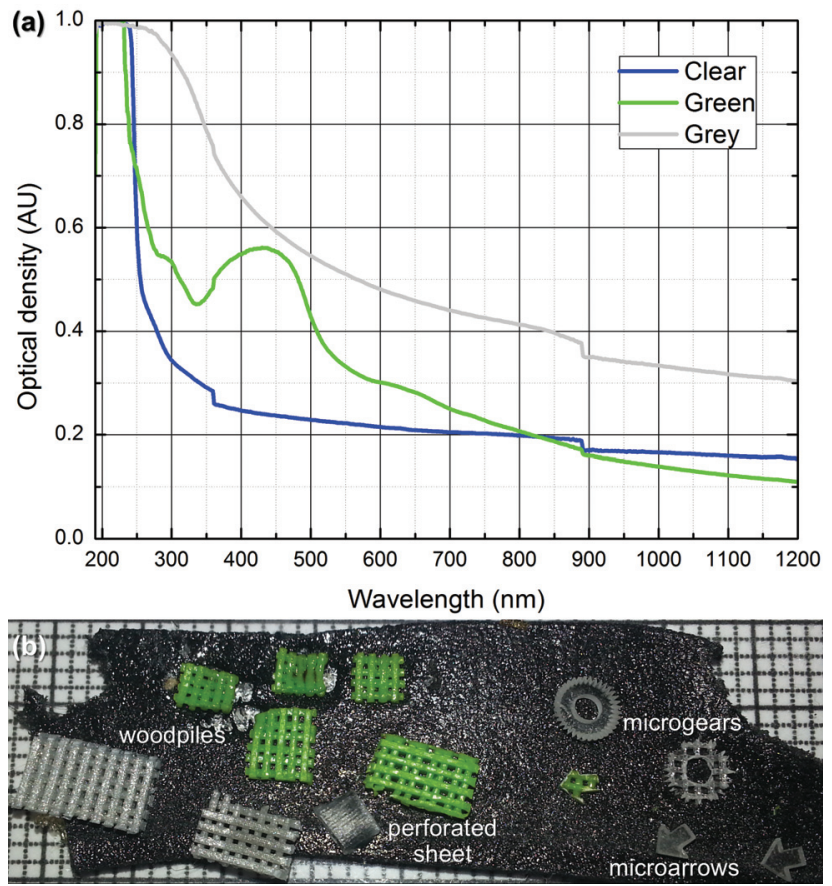

Fig. 8: (a) - measured spectra optical density of pure and colored PLA sheets (note the jump at $888 \mathrm{~nm}$ is an artifact due to the change of spectrophotometer's light source). (b) - microstructures of various shapes were processed (engraved, drilled) or obtained (cut out) via femtosecond pulse sharp beam focussing and filament assisted fabrication shown as seen by the naked eye. The reference squares seen in background corresponds to $1 \mathrm{~mm}$. 


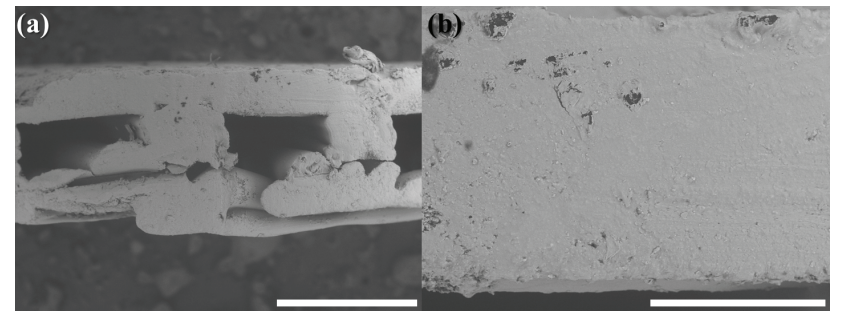

Fig. 9: (a) - a woodpile microarchitecture disk consisting of 3 layers cut out of a bigger $3 D$ microprinted sheet. (b) - a magnified side view of a single rod showing the smoothness of the FPF cutting. Scale bars are $1 \mathrm{~mm}$ and $200 \mu \mathrm{m}$, respectively.

\section{Conclusion}

In conclusion, we have experimentally implemented $300 \mathrm{fs}$ pulsed light of $1030 \mathrm{~nm}$ for the ablation of biocompatible-biodegradable PLA material. This was performed by conventionally focusing the light beam with a $\mathrm{NA}=0.3$ lens as well as introducing $75 \mathrm{TW} / \mathrm{cm}^{2}$ intensity light filament assisted fabrication. The later proposed method offers rapid fabrication and allows overcoming the common problem of accurately adjusting the focal plane as well as avoids the need of vertical translation in order to perforate plastic sheets or cut various shapes out of them. The employed method is simple and straightforward, requiring solely a sample immersion in to a water bath. Its potential applications are not limited to plastics or transparent materials, but can be used for the rapid and precise modification of ceramic, semiconductor, and metallic materials. The targeted fields of practice could be manufactured artificial scaffolds for cell culturing and tissue engineering as well as counterparts or containers micromechanics and microfluidics, giving a special concern on a 3D case.

\section{Acknowledgments}

Authors acknowledge financial support by a research grant No. VP1-3.1-ŠMM-10-V-02-007 (Development and Utilization of a New Generation Industrial Laser Material Processing Using Ultrashort Pulse Lasers for Industrial Applications) from the European Social Fund Agency. We gratefully thank Mr. E. Balčiūnas (Biochemistry Institute at Vilnius University) for the laser microfabrication sketches and Mr. E. Skliutas from Vilnius Laser University Research Center for the additional carried out experiments.

\section{References}

[1] B.N. Chichkov et al. Femtosecond, picosecond and nanosecond laser ablation of solids. Appl. Phys. A, 63(2):109-115, 1996.

[2] K. Sugioka and Y. Cheng. Ultrafast lasers-reliable tools for advanced materials processing. Light Sci. Appl., 3:435-448, 2014.

[3] R. Buividas, M. Mikutis, and S. Juodkazis. Surface and bulk structuring of materials by ripples with long and short laser pulses: Recent advances. Prog. Quant. Electron., 38(3):119-156, 2014.
[4] S. Kawata, H.B. Sun, T. Tanaka, and K. Takada. Finer features for functional microdevices - micromachines can be created with higher resolution using two-photon absorption. Nature, 412(6848):697-698, 2001.

[5] M. Malinauskas, M. Farsari, A. Piskarskas, and S. Juodkazis. Ultrafast laser nanostructuring of photopolymers: A decade of advances. Phys. Rep., 533(1):1-31, 2013.

[6] B.-B. Xu, L. Wang, Z.-C. Ma, R. Zhang, Q.-D. Chen, C. Lv, B. Han, X.-Z. Xiao, X.-L. Zhang, Y.-L. Zhang, K. Ueno, H. Misawa, and H.-B. Sun. Surface-plasmonmediated programmable optical nanofabrication of an oriented silver nanoplate. ACS Nano, 8(7):6682-6692, 2014.

[7] A. Dubietis, G. Tamošauskas, I. Diomin, and A. Varanavičius. Self-guided propagation of femtosecond light pulses in water. Opt. Lett., 28(14):1269-1271, 2003.

[8] A. Couairon and A. Mysyrowicz. Femtosecond filamentation in transparent media. Phys. Rep., 441(2-4):47189, 2007.

[9] S. Minardi, C. Milian, D. Majus, A. Gopal, G. Tamosauskas, A. Couairon, T. Pertsch, and A. Dubietis. Energy deposition dynamics of femtosecond pulses in water. Appl. Phys. Lett., 105:224104, 2014.

[10] S. Butkus, E. Gaizauskas, D. Paipulas, Z. Viburys, D. Kaskelyte, M. Barkauskas, A. Alesenkov, and V. Sirutkaitis. Rapid microfabrication of transparent materials using filamented femtosecond laser pulses. Appl. Phys. A, 114(1):81-90, 2014.

[11] S. Nolte, C. Momma, H. Jacobs, A. Tunnermann, B.N. Chichkov, B. Wellegehausen, and H. Welling. Ablation of metals by ultrashort laser pulses. J. Opt. Soc. Am. B, 14(10):2716-2722, 1997.

[12] A. Marcinkevičius, S. Juodkazis, M. Watanabe, M. Miwa, S. Matsuo, H. Misawa, and J. Nishii. Femtosecond laser-assisted three-dimensional microfabrication in silica. Opt. Lett., 26(5):277-279, 2001.

[13] J. Ihlemann, A. Scholl, H. Schmidt, and B. Wolff-Rottke. Nanosecond and femtosecond excimer-laser ablation of oxide ceramics. Appl. Phys. A, 60:411-417, 1995.

[14] K. Kuršelis, R. Kiyan, and B.N. Chichkov. Formation of corrugated and porous steel surfaces by femtosecond laser irradiation. Appl. Surf. Sci., 258(22):8845-8852, 2012.

[15] K. Kuršelis, T. Kudrius, D. Paipulas, O. Balachninaite, and V. Sirutkaitis. Experimental study on femtosecond laser micromachining of grooves in stainless steel. Lith. J. Phys., 50(1):95-103, 2010.

[16] S. Beke, B. Farkas, I. Romano, and F. Brandi. 3D scaffold fabrication by mask projection excimer laser stereolithography. Opt. Mat. Express, 4(10):2032-2041, 2014. 
[17] S. Beke, F. Anjum, L. Ceseracciu, I. Romano, A. Athanassiou, A. Diaspro, and F. Brandi. Rapid fabrication of rigid biodegradable scaffolds by excimer laser mask projection technique: a comparison between 248 and 308 nm. Laser Phys., 23(3):035602, 2013.

[18] S. Rekštytė, M. Malinauskas, and S. Juodkazis. Three-dimensional laser micro-sculpturing of silicone: towards bio-compatible scaffolds. Opt. Express, 21(14):17028-17041, 2013.

[19] R. Buividas, S. Rekstyte, M. Malinauskas, and S. Juodkazis. Nano-groove and 3D fabrication by controlled avalanche using femtosecond laser pulses. Opt. Mater. Express, 3(10):1674-1686, 2013.

[20] M. Gedvilas and G. Račiukaitis. Investigation of UV picosecond laser ablation of polymers. Proc. SPIE, 6157:61570T, 2006.

[21] K. Ratautas, G. Račiukaitis, and M. Gedvilas. Sphereto-plate glass welding using picosecond-laser radiation. J. Laser Micro Nanoeng., 8:175-182, 2013.

[22] S.D. Gittard, A. Nguyen, K. Obata, A. Koroleva, R.J. Narayan, and B.N. Chichkov. Fabrication of microscale medical devices by two-photon polymerization with multiple foci via a spatial light modulator. Biomed. Opt. Express, 2(11):3167-3178, 2011.

[23] E.T. Ritschdorff, R. Nielson, and J.B. Shear. Multi-focal multiphoton lithography. Lab Chip, 12:867-871, 2012.

[24] E. Stankevicius, T. Gertus, M. Rutkauskas, M. Gedvilas, G. Raciukaitis, R. Gadonas, V. Smilgevicius, and M. Malinauskas. Fabrication of micro-tube arrays in photopolymer SZ2080 by using three different methods of a direct laser polymerization technique. J. Micromech. Microeng., 22:065022, 2012.

[25] L. Jonusauskas, S. Rekstyte, and M. Malinauskas. Augmentation of direct laser writing fabrication throughput for three-dimensional structures by varying focusing conditions. Opt. Eng., 53(12):125102, 2014.

[26] W. Xiong, Y.S. Zhou, X.N. He, Y. Gao, M. MahjouriSamani, L. Jiang, T. Baldacchini, and Y.F. Lu. Simultaneous additive and subtractive three-dimensional nanofabrication using integrated two-photon polymerization and multiphoton ablation. Light Sci. Appl., 1:e6, 2012.

[27] R. Buividas, L. Rosa, R. Sliupas, T. Kudrius, G. Slekys, V. Datsyuk, and S. Juodkazis. Mechanism of fine ripple formation on surfaces of (semi)transparent materials via a half-wavelength cavity feedback. Nanotechnology, 22(5):055304, 2011.

[28] A. Vailionis, E.G. Gamaly, V. Mizeikis, W. Yang, A.V. Rode, and S. Juodkazis. Evidence of superdense aluminium synthesized by ultrafast microexplosion. Nature Commun., 2:445, 2011.
[29] P. Danilevicius, L. Georgiadi, C.J. Pateman, F. Claeyssens, M. Chatzinikolaidou, and M. Farsari. The effect of porosity on cell ingrowth into accurately defined, laser-made 3D scaffolds. Appl. Surf. Sci., 336:2-10, 2015.

[30] M. Farsari, A. Ovsianikov, M. Vamvakaki, I. Sakellari, D. Gray, B.N. Chichkov, and C. Fotakis. Fabrication of three-dimensional photonic crystal structures containing an active nonlinear optical chromophore. Appl. Phys. A, 93:11-15, 2008.

[31] A. Zukauskas, M. Malinauskas, L. Kontenis, V. Purlys, D. Paipulas, M. Vengris, and R. Gadonas. Organic dye doped microstructures for optically active functional devices fabricated via two-photon polymerization technique. Lith. J. Phys., 50:55-61, 2010.

[32] M. Gu, B. Jia, J. Li, and M.J. Ventura. Fabrication of three-dimensional photonic crystals in quantum-dotbased materials. Laser Photon. Rev., 4(3):414-431, 2010.

[33] M. Malinauskas, D. Baltriukiene, A. Kraniauskas, P. Danilevicius, R. Jarasiene, R. Sirmenis, A. Zukauskas, E. Balciunas, V. Purlys, R. Gadonas, V. Bukelskiene, V. Sirvydis, and A. Piskarskas. In vitro and in vivo biocompatibility study on laser 3D microstructurable polymers. Appl. Phys. A, 108:751-759, 2012.

[34] B. Farkas, A. Zsedenyi, E. Gyukity-Sebestyen, I. Romano, K. Nagy, A. Diaspro, F. Brandi, K. Buzas, and S. Beke. Excimer laser-produced biodegradable photopolymer scaffolds do not induce immune rejection in vivo. J. Laser Micro Nanoeng., 10(1):11-14, 2015.

[35] A. Žukauskas, M. Malinauskas, A. Kadys, G. Gervinskas, G. Seniutinas, S. Kandasamy, and S. Juodkazis. Black silicon: substrate for laser 3d micro/nanopolymerization. Opt. Express, 21(6):6901-6909, 2013.

[36] S. Rekštytè, T. Jonavičius, and M. Malinauskas. Direct laser writing of microstructures on optically opaque and reflective surfaces. Opt. Las. Eng., 53:90-97, 2014.

[37] C.A. Aguilar, Y. Lu, S. Mao, and S. Chen. Direct micropatterning of biodegradable polymers using ultraviolet and femtosecond lasers. Biomaterials, 26:7642-7649, 2005 .

[38] K.S. Tiaw, S.W. Goh, M. Hong, Z. Wang, B. Lan, and S.H. Teoh. Laser surface modification of poly(ecaprolactone) (PCL) membrane for tissue engineering applications. Biomaterials, 26:763-769, 2005.

[39] Y. Lu and S.C. Chen. Micro and nano-fabrication of biodegradable polymers for drug delivery. Adv. Drug Deliv. Rev., 56:1621-1633, 2004.

[40] K. Stolberg, S. Friedel, B. Kremser, and M. Roehner. IR and green femtosecond laser machining of heat sensitive materialsfor medical devices at micrometer scale. Proc. SPIE, 8968:89680E, 2014. 
[41] D. Gomez, I. Goenaga, I. Lizuain, and M. Ozaita. Femtosecond laser ablation for microfluidics. Opt. Eng., 44(5):051105, 2005.

[42] M. Malinauskas, S. Rekštytè, L. Lukoševičius, S. Butkus, E. Balčiūnas, M. Pečiukaitytè, D. Baltriukienè, V. Bukelskienė, A. Butkevičius, P. Kucevičius, V. Rutkūnas, and S. Juodkazis. 3D microporous scaffolds manufactured via combination of fused filament fabrication and direct laser writing ablation. Micromachines, 5:839-858, 2014.

[43] S. Butkus, D. Paipulas, R Sirutkaitis, E. Gaižauskas, and V. Sirutkaitis. Rapid cutting and drilling of transparent materials via femtosecond laser filamentation. J. Laser Micro Nano Eng., 9(3):213-220, 2014.

[44] P.E. Petrochenko, J. Torgersen, P. Gruber, L.A. Hicks, J. Zheng, G. Kumar, R.J. Narayan, P.L. Goering, R. Liska, J. Stampf, and A. Ovsianikov. Laser 3D printing with sub-microscale resolution of porous elastomeric scaffolds for supporting human bone stem cells. Adv. Health Mat., page 10.1002/adhm.201400442, 2015 .

[45] A.C. Scheiwe, S.C. Frank, T.J. Autenrieth, M. Bastmeyer, and M. Wegener. Subcellular stretch-induced cytoskeletal response of single fibroblasts within 3D designer scaffolds. Biomaterials, 44:186-194, 2015.
[46] D. Wu, L. Niu, S. Wu, J. Xu, K. Midorikawa, and K. Sugioka. Ship-in-a-bottle femtosecond laser integration of optofluidic microlens arrays with center-pass units enabling coupling-free parallel cell counting with $100 \%$ success rate. Lab Chip, page 10.1039/C4LC01439A, 2015.

[47] J. Maciulaitis, M. Deveikyte, S. Rekstyte, M. Bratchikov, A. Darinskas, A. Simbelyte, G. Daunoras, A. Laurinaviciene, A. Laurinavicius, R. Gudas, M. Malinauskas, and R. Maciulaitis. Preclinical study of SZ2080 material 3D microstructured scaffolds for cartilage tissue engineering made by femtosecond direct laser writing lithography. Biofabrication, 7:015015, 2015.

[48] A. Žukauskas, G. Batavičiūtè, M. Šciuka, T. Jukna, A. Melninkaitis, and M. Malinauskas. Characterization of photopolymers used in laser 3D micro/nanolithography by means of laser-induced damage threshold (LIDT). Opt. Mat. Express, 4(8):1601-1616, 2014.

[49] A. Zukauskas, G. Bataviciute, M. Sciuka, Z. Balevicius, A. Melninkaitis, and M. Malinauskas. Effect of the photoinitiator presence and exposure conditions on laserinduced damage threshold of ORMOSIL (SZ2080). Opt. Mat., 39:224-231, 2015.

(Received: July 3, 2014, Accepted: April 13, 2015) 